\title{
Alien presence in the home: the design of Tableau Machine
}

\author{
Mario Romero · Zachary Pousman · \\ Michael Mateas
}

Received: 19 December 2005 / Accepted: 19 September 2006

(C) Springer-Verlag London Limited 2007

\begin{abstract}
We introduce a design strategy, alien presence, which combines work in human-computer interaction, artificial intelligence, and media art to create enchanting experiences involving reflection over and contemplation of daily activities. An alien presence actively interprets and characterizes daily activity and reflects it back via generative, ambient displays that avoid simple one-to-one mappings between sensed data and output. We describe the alien presence design strategy for achieving enchantment, and report on Tableau Machine, a concrete example of an alien presence design for domestic spaces. We report on an encouraging formative evaluation indicating that Tableau Machine does indeed support reflection and actively engages users in the co-construction of meaning around the display.
\end{abstract}

Keywords Design for enchantment - Computer vision . Ambient information systems - Generative art .

Formative evaluation

\section{Introduction}

With the success of the research agendas of Ubiquitous Computing (UbiComp) and Ambient Intelligence (AmI), computing has left the desktop. However, much of this

M. Romero $\cdot$ Z. Pousman $(\bowtie) \cdot$ M. Mateas

Georgia Institute of Technology, TSRB, 85 th st. NW, Atlanta,

GA, USA

e-mail: zach@cc.gatech.edu

M. Romero

e-mail: mromero@cc.gatech.edu

M. Mateas

e-mail: michaelm@cc.gatech.edu work still retains the dominant "information access and task-support" paradigm from desktop interaction, constraining episodes of computer use to hours and minutes rather than weeks and months, assuming deployed systems will be used to support well-defined, narrowly construed tasks, and focusing on task-based success metrics. Our work seeks longer-term interactions with systems not predicated on a task support model nor evaluated in terms of optimal performance. Instead, we propose a kind of interaction typified by long-term pleasurable experiences of contemplation and reflection. The computer, imbued with AI-based perception, interpretation, and artistic output becomes another entity in the environment. It achieves a social presence by sensing and reacting somewhat unpredictably to the behavior of humans. We call this approach alien presence to convey the idea that we create a nonanthropomorphic social entity [1]. An alien presence is an enchanting artifact that opens new perspectives on the familiar and everyday. Its alien subjectivity provides users with novel reflections of the social situation in which the alien presence is embedded.

This paper introduces the alien presence design strategy and describes Tableau Machine, an alien presence for the home. Human-computer interaction and UbiComp researchers have become increasingly interested in analyzing domestic space and home life. Analyses of home life by researchers such as Dourish [2] and Crabtree [3] inform our computational approach for characterizing domestic activity.

\subsection{The enchanting alien}

The goal of Tableau Machine is to bring enchantment to domestic settings through the design strategy of alien 
presence. Recent work by McCarthy et al. [4] has opened a conversation on the attributes contributing to enchanting experiences with technology. Two primary attributes they identify are emotional attachment and a lack of perfect understanding. Paraphrasing Bennett, they say the "combination of emotional attachment together with a sense of something not yet understood leaves us feeling disrupted but also alive, attentive, and curious." They also note that the novelty of the experience, its being outside the everyday, "awakens us to wonder and to the wonder of life..." As a design strategy for achieving enchantment, alien presence combines elements of both ambient intelligence and ambient information displays, while questioning and changing assumptions made by both fields.

Much work in ambient intelligence attempts to infer detailed and concrete models of human intentionality, and use these models to proactively intervene in user activities. Such systems require robust activity recognition, that is, the classification of sensor data into concrete activity categories. Since the goal of Tableau Machine is to awaken curiosity and support reflection about everyday activity, it must characterize and respond to the subtle but rich social and cultural aspects of human activity without committing to inferences about exactly what activities are being performed and without judgment on any social or cultural configuration. Unlike activity recognition, which models discrete and concrete activities and states such as "cooking" and "cleaning", or emotional labels such as "happy" and "fighting," we characterize abstract qualities of activity. We designed Tableau Machine to give a sense of social mood of the home, such as "togetherness" and "busyness." In contrast to activity recognition, we call this approach activity characterization. By taking enchantment as a first class design goal and focusing on AI-based responses to the qualities of social activity, we are able to identify site-specific features dynamic rhythms of social environments, sidestepping the need for AI-complete systems (systems that accurately and completely model the whole of human activity). Unlike activity recognition, which, to work robustly in rich environments, requires a human-level understanding of action and context, activity characterization makes use of site-specific proxy measures that stand in for inaccessible features of a cultural context (in our case, the home).

The focus on enchantment also impacts our design of the representations that serve as outputs for the Tableau Machine. Work in ambient information displays often makes use of relatively transparent mappings between simple data values and the display. The goal is to produce decodable displays, where users can "read through" the sensory display to a qualitative value (range) on one or more data streams (e.g., downtown traffic is light, many people are in the office, a particular stock's price is high).
In contrast, alien presence systems actively interpret incoming data streams and use this active interpretation to build non-transparent data displays. By avoiding simple one-to-one mappings between data and display, the user has more space to engage in co-interpretation, that is, for the system to serve as a provocation rather than an information pipe. If done correctly, users should have the sense of the system as an independent, non-human subject, who has its own interpretation of the activity engaged in by the user; living with such a system long-term becomes a process of getting to know the alien subjectivity of the system, and of actively constructing meanings relating the alien interpretation to lived experience.

As a design strategy alien presence achieves enchantment both through the alien perspective it provides on daily life, and by being an active, always available presence within daily life. The move away from activity recognition towards activity characterization creates abstract and ambiguous interpretations of daily life. Combined with a complex, non-one-to-one display mapping, this creates the sense of a not-immediately understood, yet eventually understandable system. By hopefully sustaining a rich space of interpretation that supports users in reading the system as an alien subject, we create the sense of an active presence, a magic object that knows something about the user, has responses to this knowledge, and thus supports a long term emotional engagement.

As our investigation into alien presence progressed, we sought feedback from people engaged in everyday domestic situations. We report throughout the paper on a formative evaluation of Tableau Machine. This formative evaluation did not involve people living long-term with Tableau Machine, but rather consisted of a single evening of social activity similar to a dinner party with six adult participants (two male) cooking, eating, and retiring to the living room for dessert. During this event, Tableau Machine presented its interpretations as compositions on a screen in the living room (see Fig. 1). The participants were a group of friends who all knew each other prior to the study, coming from a variety of walks of life. None of them were technologists, and were thus not predisposed to taking an unusual level of interest in or possessing many assumptions about how Tableau Machine functions behindthe-scenes. The evaluation was not intended to be definitive, but rather to provide us with interim feedback on the Tableau Machine implementation of the alien presence strategy.

\section{Related work}

Although usability and utility have been the conventional research themes within $\mathrm{HCI}$, a wider set of concerns, such 


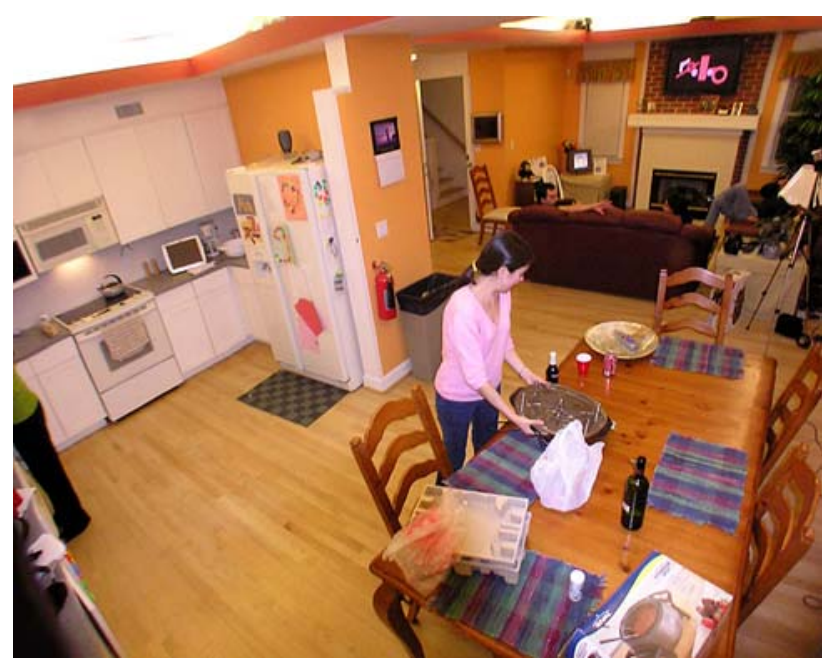

Fig. 1 Tableau Machine output over the chimney in the living room of the Aware Home during a social night with six participants of a formative evaluation

as enjoyment, aesthetics, wonder, and engagement, are emerging [5]. Traditional usability design and evaluation methodologies do not directly transfer to these novel topics. Practitioners have begun to explore new methodologies for design and evaluation. For example, Höök et al present the design of an evaluation methodology for Influencing Machine, a child-like drawing system [6]. One of the authors' main conclusions is that the evaluation of interactive art systems should help the artists who create such systems to understand how users interpret their systems. Their goal is to give artists a "grounded feeling for how the machine was interpreted and the message was communicated." Note that this is a different evaluation outcome than is produced by traditional user centered techniques.

In "Windows and Mirrors", Bolter and Gromala argue for understanding the computer interface both as a transparent window for accessing data and operations, and as a reflective mirror for contemplating the medium itself [7]. They present several examples of explorations of the computer as a medium. Wooden Mirror by Rozin and Text Rain by Utterback are notable examples of using the computer as a medium for experience, contemplation, and reflection.

We consider Tableau Machine to be an instance of Expressive AI in general and generative and interactive art in particular. Expressive AI, introduced by Mateas [8], proposes that by simultaneously treating the design and evaluation issues in art practice and advances in artificial intelligence and as first class research questions, new research agendas are opened up in both AI and humancomputer interaction. Examples of Expressive AI systems are Office Plant \#1 [9], Petit Mal [10], Giver of Names [11], and Façade [12]. Some examples of generative art systems are Cohen's Aaron [13] and Lioret's being paintings [14]. Further art systems interact with viewers of the work, making a viewer into a performer. Examples include Interactive Wallpaper [15], Utterback's Untitled 5 [16], and Artifacts of the Presence Era [17].

We also take inspiration from Ambient Information Systems [18], an area of research into decorative artifacts that present data, such as weather forecasts, email traffic, and stock market prices, in an ambient manner with minimal interruption to primary tasks. Some exemplars in this area include the Ambient Orb and Water Lamp of Ihsii's Tangible Media Group [19], and the Informative Art [18] system. These systems map data to a visual representation using relatively simple mappings. For example, the Ambient Orb can map a stock market index to colors as a function of how far up or down the index is over the trading day. Tableau Machine departs from this singular mapping between data and representation. Instead, we seek to produce ambiguous representations with multiple "correct" mappings back to the source data.

\section{Overview of the system}

Tableau Machine is deployed in the Aware Home, Georgia Tech's Ubicomp living laboratory [20]. Figure 2a shows its floor plan with overhead images and place boundaries.

The system uses the camera system in the Aware Home as a motion detector, immediately discarding the video frames. Thus, we obviate privacy concerns, while achieving motion detection at a square inch resolution. We divide space into socially meaningful zones and use the motion within zones to compute three proxy measures of the mood in the environment: Social Energy, Density, and Flow.

The system maps the proxy measures to design attributes, such as direction and color, of two-dimensional visual compositions; generated compositions are displayed on a screen in the living room (see Fig. 1). By non-deterministically mapping our proxy measures to abstract attributes, we avoid simple one-to-one correspondences between activity and individual composition elements. Instead, the compositions give an overall sense of the mood of the home. The output changes every few minutes.

\section{Characterizing activity}

Activity can be characterized by the motion generated while performing it and by the place in which it occurs with sensitive measuring instruments and over time, all human activity produces motion, even those activities not traditionally considered physical. Unfortunately, standard motion detection technologies are insensitive to smaller 
Fig. 2 a Floor plan with overhead images and Semantic Activity Zones. b Adjacency graph
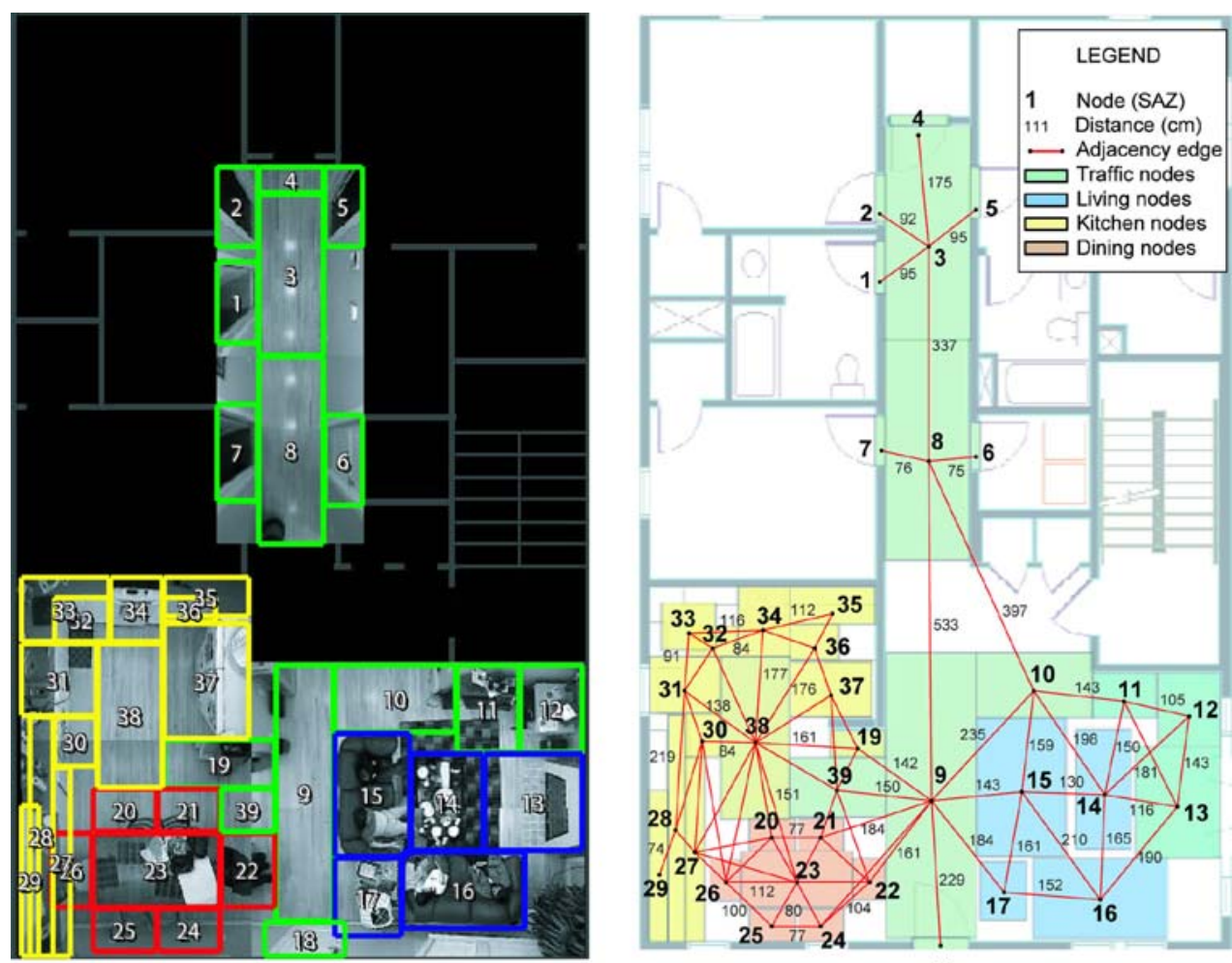

18 movements. With computer-vision-based techniques we are able to detect quieter motion, such as scratching, fidgeting and small shifts of weight.

Locations provide spatial and social context for activity $[2,21]$. We divide the public spaces of the home into four areas of activity: transit, kitchen, dining, and living room areas. Each area is further subdivided into socially meaningful and spatially stable regions of the home around objects such as doors, cabinets, halls, and furniture (see Fig. 2a, b). We refer to these subdivisions of space as Semantic Activity Zones (SAZ). Simple and robust adjacent frame difference computations [22] provide highresolution motion measurements, which in turn provide the activity level in each SAZ.

\subsection{Social energy, density and flow}

To uncover activity patterns, we conducted studies of the use of space in the Aware Home. We observed groups of participants engaged in natural activity, analyzing several different situations using grounded theory methodology [23]. To facilitate qualitative analysis we built a visualization tool called the Activity Table (see Fig. 3). The rows of the table encode space and the columns, time. The cells are dark when activity is low and bright when it is high. Figure 3 shows activity patterns over the course of a dinner party. Some activities can be discriminated from others based solely on aggregate motion. For example, "eating raclette" is more active than "eating dessert" even though both are predominantly in the dining areas. Other activities can be differentiated by the paths they leave over the SAZs. For example, people setting up the game board cross multiple SAZ boundaries while people playing the game generally remain in a single SAZ; the same holds for cooking and setting the table versus eating. Based on our activity table analysis, we developed three proxy measures of social activity in the home: social energy, density, and flow.

To operationally define our proxy measures, we organize the SAZs into a graph structure (see Fig. 2b). Each node represents the physical center and area of a SAZ. Adjacent nodes in the graph correspond to physically adjacent SAZs in the home. Edge weights in the graph correspond to physical distances.

Social energy is motion aggregated over a place during a period of time. To determine the social energy at place $\mathrm{P}$, we sum motion pixels over the group of semantic activity zones belonging to $P$ over some period of time. We compute social energy in each SAZ, and then compute energy in the kitchen, dining room, living room, transit areas, and the entire home. Social energy provides a proxy measure of the level of activity. Energy will rise with larger or faster movement, or with more people moving.

Social density is a proxy measure for "togetherness", how physically close people are in the environment. Formally, we define social density as the energy across all 
Fig. 3 Activity table: rows encode space (SAZs), columns encode time, and brightness encodes activity level. Highlighted areas show activities recognized by observing the original video

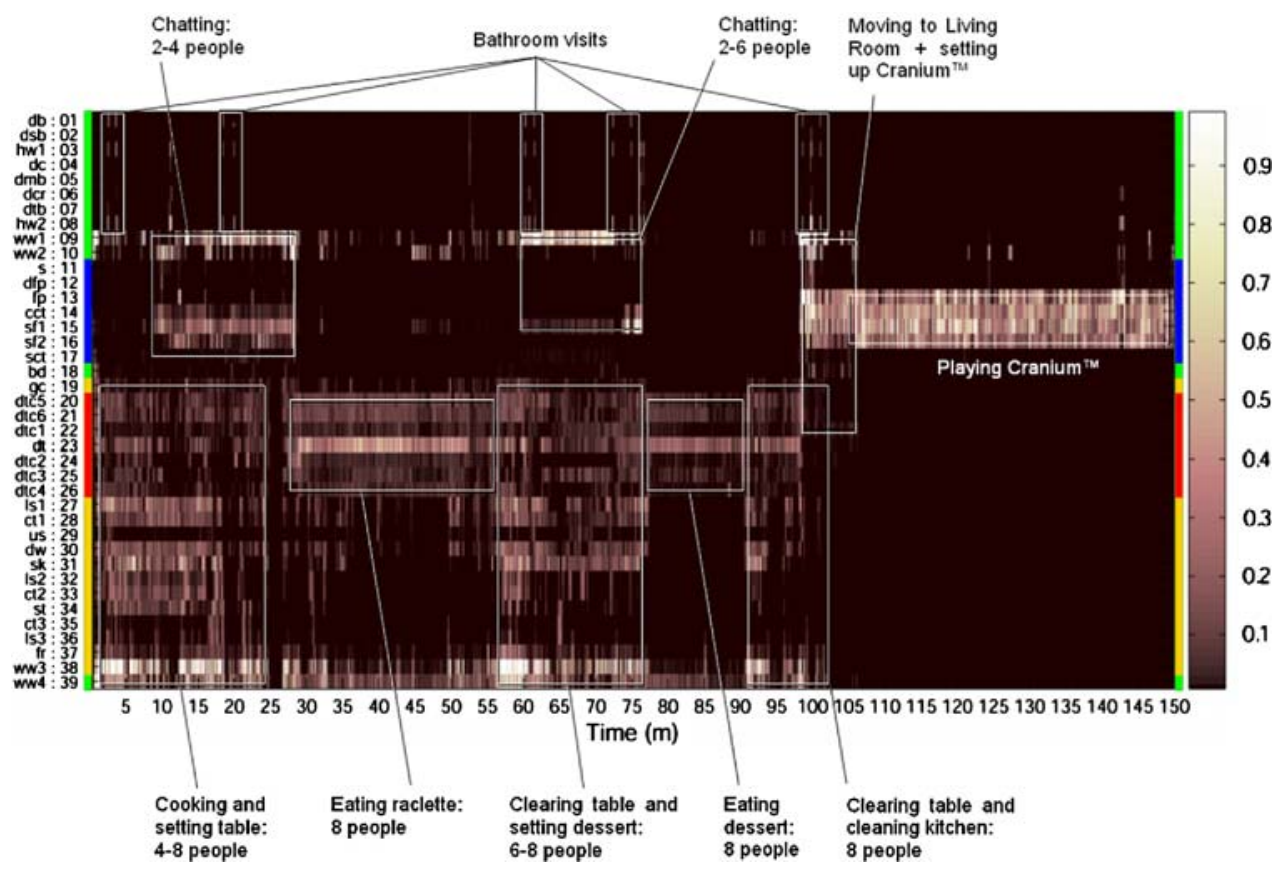

active SAZs divided by the graph distance connecting all active SAZs. A historically computed threshold determines which SAZs are active. Density tells us whether energy is densely packed, or spread out through the home.

Social flow captures the transfer of energy between adjacent SAZs. Formally, we say that there is social flow from adjacent $S A Z Q$ to $R$ at time $t$ if the derivative of the energy in $\mathrm{Q}$ with respect to time is negative and $\mathrm{R}$, positive. This gives us a flow matrix for all the edges in the adjacency graph. While social flow is most obviously generated by people moving across SAZs, it is also generated by more subtle phenomena such as reciprocal talking and gesturing across adjacent SAZs.

\subsection{Discussion of social activity measures}

Density is our working approximation of togetherness. We observed that density is highly correlated with togetherness, but togetherness is not always a consequence of density. People can be packed together without being together. For example, while clearing the table, people may be crowded between the kitchen and the table, but each one is doing their own task, paying only sporadic attention to others. We can say there is high density but low togetherness. If we include social flow, we can differentiate this activity from, for example, having dinner, which has high density, low flow, and high togetherness. Thus, flow and density together can explain the social concept of togetherness better than density alone.

Similarly, a social descriptor like "busyness" grounds out in our analysis as a combination of energy and flow.
For example, we observed two instances of high energy in the living room. In the first instance, eight friends were playing Cranium ${ }^{\mathrm{TM}}$. The game has several very physical activities, like acting, drawing and sculpting. Furthermore, laughter while playing the game generates a lot of in-zone movement, which accumulates over time and appears as social energy. However, in the second instance, when the group put away the game and cleaned up the living room, they also generated in-zone movement and energy, though the activities are socially very different. Flow helps to segregate the two types of activities. While playing the game people remained within a single SAZ, or had smaller inter-zone flows, occupants clearing the living room moved between SAZs numerous times creating higher flow values.

However, even with all three measures, many household activities are aliased. They produce nearly identical energy, density, and flow measures, though the actual activities are different. For example, an activity "having a party" may create similar data to activity of a "household fight." This mismatch or aliasing contributes to our ultimate goal of reflecting an alien interpretation of domestic life. For our alien, having a party and having a fight are both instances of highly excited, dense activity. The goal with our proxy measures is to create intelligible interpretations of activity, while providing room to provide their own interpretations of system activity. We seek to give users a novel window onto their own activity, and a sense of Tableau Machine as an alien social being with its own idiosyncratic interpretation of the household.

In our formative evaluation, the participants found correlations between their activity and the system's compositions, but did not learn exactly how their activities 
were influencing the machine. This is a positive result; if participants had been able to quickly interpret the underlying mechanics of the system, it would lose its enchanting qualities. Here we present two examples of how participants fabricated simple theories about the system's workings. While participants cooked at the table (a raclette), $\mathrm{P}$ remarked: "Hey, now it's all red!" pointing to the newest composition. N replied: "It's because I'm burning everything. That explains it!" Later, N said to A: "Ah! Have you seen that?" as she pointed to the composition from the kitchen sink. A replied: "That's pretty! And why did it change like that?" $\mathrm{N}$ : "Because we're washing the dishes."

A second spontaneous theory that rose during the experience was a conception of "social balance." After dinner, the women began to clean up while the men retired to the living room. Participant A (woman) exclaimed "Hey! We had to clear the table." M (man) replied "Well, the system was unstable. If there is too much movement in the kitchen... um [laughter]...I am concerned [the system] might collapse." This interaction, while a humorous one, can be seen as a brief moment of self-reflection on a common social trope. It also evidences the type of engagement and stories people may build around the machine.

As designers, it was quite valuable to observe how participants engaged in co-interpretation during the formative evaluation. Participants had socially mediated labels for their activities, such as "washing the dishes," and had in mind the kinds of representations that might (metaphorically) lead from them. Participants assumed the machine's compositions had some interpretation of their activity. Though the system output is in fact not based on recognition of specific activities, such as washing the dishes, participants were willing and able to interpret the system output in terms of their concrete activities. This provides evidence that our three proxy measures can support useful and pleasurable readings.

\section{Visual output}

The energy, density, and flow measures are used by the art generator, the final stage of Tableau Machine. The display serves as the mouthpiece of the system, producing statements about its alien and subjective interpretations of human activity. The display is not intended to provide an immediately readable visualization of some underling data stream, but to challenge, to invite questions, to make strange everyday activity. This process of adopting a new stance on a familiar activity has been characterized by and Shklovsky and later by Bell et al. as "defamiliarization" [24, 25].

Our goal as designers is to achieve intelligibility without predictability. Simple one-to-one mappings between data and display would inhibit the richness of the experience and prevent occupants from seeing the system as intelligent. A direct mapping, for example a representation where people are colored dots and they bounce around inside a box representing a home, gives the feeling not of wonder and alien intelligence, but of surveillance and rote regurgitation. The other extreme, a fully random mapping between activity and visual output, also fails to enchant; instead it is unintelligible. We seek a mid-point along this spectrum between one-to-one and random mappings. We posit the best mappings are those that give a data-driven experience, without exposing specific values. We strive for the system to be intelligible and interpretable, without the user being able to fully predict or explain the system.

Note that the individual images produced by the art generator are not intended in themselves to produce enchantment. Rather, it is the constantly changing, dynamically generated sequence of images, and the user's construction of meanings relating the images to activities in the home, which create an enchanting experience. That is, what enchants is not an individual static image, but rather a "living painting", which knows something about household activity and reflects this knowledge in constantly changing imagery.

\subsection{Co-interpretation}

We define co-interpretation as the process by which both the user and the machine assign meaning to the display. The user endows the display with meaning from her personal perspective. The novelty of an alien presence is that it is driven by algorithmic simulation of interpretative processes lending themselves to user recognition of awareness. In other words, the human spectator is able to perceive the machine as an active meaning-making presence. The user interprets the machine's interpretation of the user's activity, which forms a cycle of co-interpretation.

The artist/programmer defines the meaning-producing mechanisms of the machine through their architectural and algorithmic choices. In particular, we built several layers of interpretation into Tableau Machine. First, it senses motion. Next, it integrates localized motion into social energy, density, and flow. Finally, the social activity dynamics modulate a generative art system; the sensed dynamics influence the "flavor" of the image by changing composition parameters. Tableau Machine and the spectator are both active meaning-assigning agents, coupled in a process of co-interpretation.

The spectator of an alien presence does not actively interact by pushing buttons or otherwise explicitly driving its activity. Rather, her unconscious daily activities cause perceptions in the machine that drive its artistic compositions. It is this level of unconscious activity that is reflected 
to the user. The system provides a novel perspective on the mundane and everyday. The user interprets the state of the system based on her understanding of how the system produces compositions, an understanding which includes her own projected meanings and narratives. This process of interpretation takes place over a prolonged period of time, as the user begins to learn regularities between household activity and system output.

\subsection{Compositions}

Tableau Machine generates a series of 2-dimensonal compositions in response to the sensed social activity dynamics. For the formative evaluation, we used a stub version of the composition generator in which the system selects a composition to display from a large fixed set of compositions, selecting one every five minutes as a function of the sensed energy, density and flow. The compositions differ from one another in color scheme, form, and shape selection. Selecting from a pool of handcreated 2-dimensional compositions and experimenting with different mappings allowed us to validate that our compositions and mappings support provocative user interpretations before investing energy in the composition generator. Participants' reactions to the compositions (and implicitly, to the mappings from sensed data to composition features) during the formative evaluation is informing our current work on the fully generative output system.

Tableau Machine's compositions are design riffs on a quote by LeCorbusier: "a house is a machine for living in." The quote, while steeped in the modernist tradition that regards life as the workings of a machine, is an interesting launchpad for our work. It inspires questions: If a house is a machine, what does it look like? What fuel does it consume? What does it produce? Our compositions are iconic views of machines, with gears, belts, and rectangular bars (see Fig. 4).

We compose the primitive shapes according to the language of graphic design, drawing guidance from graphic design principles. The selection (for the formative evaluation) and generation (current work) of compositions is organized around the following design principles: dominance, direction, number of shapes, shape selection, and color palette.

\subsection{Mapping}

When creating the set of compositions for the formative evaluation, we started with a preliminary mapping between sensed data and visual output. These suppositions were reinforced and honed as we created sample compositions and varied the design elements.

\subsubsection{Dominance}

Some compositions are made of large shapes that hold the focus of the composition. Others have shapes more evenly distributed with multiple focus points (though not excessively so, since dominance helps create visual interest). Compositions with more dominance connote higher social density in the home.

\subsubsection{Direction}

Each composition has a direction: horizontal, vertical or oblique. Oblique compositions suggest motion, and are
Fig. 4 Example renderings of Tableau Machine
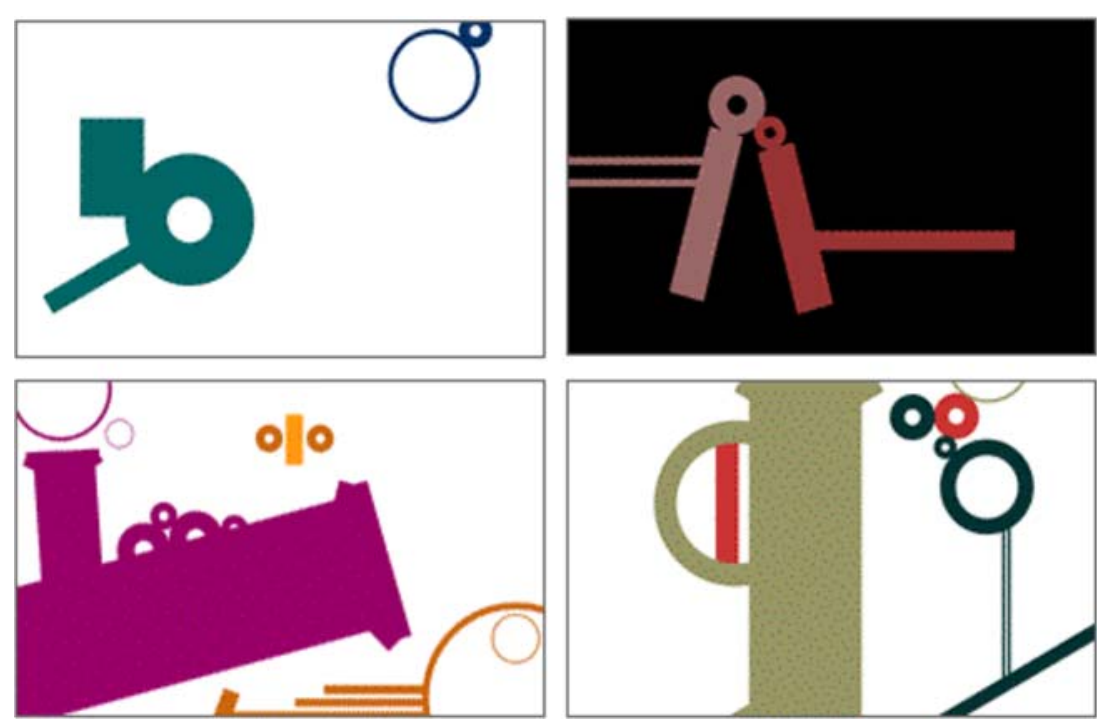
thus used to connote higher levels of activity (social energy).

\subsubsection{Number of shapes}

Some compositions have as few as 4 primitive elements. Others have 20 or more. A large number of shapes translates into compositions with more visual delicacy, which connotes higher social flow. Compositions with many shapes are selected when the social flow is above a threshold value.

\subsubsection{Shape selection}

When social energy is high, more circles are included in the compositions. Further, as energy rises, more obliquely angled rectangles are drawn.

\subsubsection{Color}

Four-color palettes are used; one is cool, another quiet, a third energetic, and a fourth bold. We selected these palettes from a graphic design color index [26]. The bold and energetic palettes are used when the system detects high energy and more quiet palettes when energy is below a threshold value.

In our evaluation we sought to distinguish between denotative effectiveness, our ability to convey a sense of mechanical or machine-like scenes, and connotative effectiveness, our ability to convey the flavor of social activity in the home. The idea of representing machine parts was understood and quickly assimilated. Participants claimed compositions looked like "a machine", "a pipeline" and the circular shapes looked like "gears." Some participants liked this composition space; others wished for more naturalistic designs, specifically stating that the system was "too rigid" and needed "more curved" shapes. Participants did not have an easy time understanding the connotative meanings of the compositions, that is, the qualitative similarity in activity that lead to similar compositions. Since our design goal is that users' interpretation of compositions produced by the system will slowly develop over time, it is not surprising that in the course of a single evening (the time span of the formative evaluation) participants were not able to develop sophisticated connotative readings of the compositions. In a short exposure to the system, the best that can be hoped is that participants will actively engage in forming hypotheses and telling stories about the system. This did in fact occur during the formative evaluation. In a planned longitudinal study, we will explore whether and how participants form longerterm, more sophisticated connotative readings.

Participants complained Tableau Machine did not show enough different compositions. Since the formative evaluation took place over a single evening at a dinner party, the compositions were almost exclusively from the most active region of the composition space. Without a generative composition system in place, a relatively small number of compositions were shown over the course of the evening. Eventually participants stopped trying to interpret compositions, remarking "oh, that one again." The quick recognition of identical compositions confirmed the need for a fully generative composition system in order to elicit more interest and wonder about the system over extended periods of interaction.

Further, participants did not find the active compositions kinetic enough and the calm compositions subdued enough. Participants saw some relationship between calm compositions and calm spans of time, but this sense was not strong. Their perceptions are due in part to the restricted part of the composition space that was presented during the dinner party, but we also found the system needs to produce compositions more frequently. A new composition every five minutes may be enough for a long-term installation, but during a single evening, participants wanted the Tableau Machine to react more rapidly. We are exploring the use of more rapid cycles of generation during "active" times in the house and slower cycles of composition generation during calmer moments. Another element we are exploring in order to bring more activity to the compositions is the judicious use of animations in each composition (and possibly animation between compositions).

\section{Discussion}

In this paper we have presented the alien presence design strategy and described Tableau Machine, a concrete example of this strategy. Based on our design experience and theoretical investigations surrounding Tableau Machine, as well as feedback from our formative evaluation, we have identified the following design guidelines for enchanting alien presence systems: use proxy measures to characterize rather than recognize activity, use data-driven visualizations that avoid one-to-one mappings that directly expose underlying data streams, design for co-interpretation, and design so as to resist mechanical, system-level understanding. We discuss each in turn, relating the guideline to our experience with Tableau Machine.

Alien presence requires new ways of transforming and interpreting sensor data. In a departure from AI and computational perception research, such systems do not attempt to recognize discrete human activities nor model the 
intentions of humans but rather to characterize activity. For Tableau Machine, proxy measures for togetherness, activity, and flow create a space for characterizing qualitative differences in home activity. Characterization gives us an operational definition of social mood more robust than activity recognition. Furthermore, even if we had started with activity recognition approaches, it is unclear how one would build up a general sense of social mood from atoms of recognized activity.

Unlike ambient information displays which typically employ simple mappings between data and representation, alien presence systems seek to inhabit a middle space between simple one-to-one mappings and incomprehensible chaos; enchantment lies in this middle space. In the Tableau Machine display we achieve this by mapping computed proxy measures to the design attributes of the $2 \mathrm{D}$ composition, rather than to individual elements of the composition. The feedback from our formative evaluation shows that Tableau Machine makes progress toward this design goal. Participants invented explanations for how their activities changed the displayed composition. However, participants were quick to notice identical compositions, and thus to loose interest in the ongoing process of co-interpretation. To be successful over the long term, an alien presence must be generative, creating nonrepeating displays, even for the same sensed data. Mapping data (or computed proxy measures) to visual design attributes rather than individual elements aids in the construction of an enchanting display designed for longterm enjoyment and engagement.

Alien presence seeks to provide co-interpretation, where the system interprets users and users interpret the system. Participants were asked directly during the focus group about where, if it existed at all, the "art" was in Tableau Machine. Different participants had differing views of definitions of art and gave disparate examples of art media that Tableau Machine resembled including paintings and installation works. One participant came to the notion that they themselves were the art, or at least a part of it, and some of the other participants agreed with her. The participants saw themselves as integral parts of the installation, one remarking she was "painting by behaving." Participants interpreted the system, and their comments hinted they saw the system as an observing entity. In a planned longitudinal study, we will explore if and how the sense of co-interpretation develops as the occupants begin to ascribe knowledge level understandings to the system.

Users should develop an understanding of an alien presence at the level of stories about its interpretations, thoughts and desires, instead of at the level of routines, functions, and data. Allan Newell contrasts knowledge level understanding, understanding an entity at the level of thoughts and intentions, with system level understanding, understanding what makes an entity work at the code (or biological) level [27]. Humans have knowledge level understanding of one another. An alien presence should be readable by observers at the knowledge level, and not at the level of sensing algorithms and interpreted values of computed measures. Knowledge level understanding creates an active sense of presence, which helps maintain enchantment. It is hard to make strong claims about Tableau Machine at this point regarding whether evaluation participants are interacting with the system at the knowledge level or at the system level. From the formative evaluation, we sense that we are on the right track towards creating a system that is understood as knowing, seeing, and thinking thoughts about activity in the home. Participants did not immediately identify the system's sensing modes or interpretations, nor the specifics of the composition selection algorithm.

\section{Future work}

Future work on Tableau Machine is proceeding on multiple parallel tracks. We are building a rule-based generative art system based on feedback from the formative evaluation. The generative system accepts values for composition features (e.g., a level of dominance, direction, color palette, etc.) and stochastically generates a composition with these features. Though the collection of hand-produced compositions was a successful first step in conveying the flavor of activity in the home, the repetition of compositions interfered with co-interpretation, even over the course of a single evening. Additionally, we will also tune the system to produce compositions more frequently, which will give users faster feedback and a chance to develop more complex stories about the system's inner-workings. Finally, we will further refine our notion of activity characterization so as to make this a design resource available to other designers of enchanting systems.

We are also preparing for a longitudinal evaluation. We are making Tableau Machine portable so it can be installed in the home's of study participants. Over the course of five weeks, we will evaluate the long-term interactions people have with the system, and how their perception of it changes over time. We will probe participant's understanding of the machine and whether a coherent knowledge-level understanding develops.

In the longer term, we are interested in building a robotic, sculptural Tableau Machine. We suspect a three dimensional work will be more evocative and more readily considered as an alien intelligence in the home. Eventually we will move outside of the home environment to other spaces that are ripe for defamiliarization and have their 
own distinct social character, such as outdoor public spaces, sports arenas, bars, and offices [9]. Though these spaces are qualitatively different, we would like to test activity characterization over a wider range of human experiences.

\section{References}

1. Mateas M, Romero M (2005) A preliminary investigation of alien presence. In: Proceedings of human-computer interaction international

2. Dourish P (2001) Where the action is: the foundations of embodied interaction. The MIT Press, Cambridge

3. Crabtree A, Hemmings T, Rodden T (2002) Pattern-based support for interactive design in domestic settings. In: Proceedings of the conference on designing interactive systems: processes, practices, methods, and techniques. ACM, London

4. McCarthy J, Wright P, Wallace J, Dearden A (2005) The experience of enchantment in human-computer interaction. Personal Ubiquitous Comput. doi:10.1007/s00779-005-0055-2

5. Blythe MA et al (2003) Funology: from usability to enjoyment Human computer interaction series, vol 3. Kluwer, Boston

6. Höök K, Sengers P, Andersson G (2003) Sense and sensibility: evaluation and interactive art. In: Proceedings of the SIGCHI conference on human factors in computing systems. ACM, Ft. Lauderdale

7. Bolter J, Gromala D (2003) Windows and mirrors: interaction design, digital art, and the myth of transparency. The MIT Press, Cambridge

8. Mateas M. (2001) Expressive AI: a hybrid art and science practice. Leonardo J Int Soc Arts Sci Technol 34(2):147-153

9. Boehlen M, Mateas M (1998) Office Plant\# 1: intimate space and contemplative entertainment. Leonardo J Int Soc Arts Sci Technol 31(5):345-348

10. Penny S (1997) Embodied cultural agents at the intersection of robotics, cognitive science and interactive art. In: Working notes of the socially intelligent agents symposium. AAAI, Menlo Park

11. Huhtamo, E. (1998) Silicon remembers ideology, or David Rokeby's meta-interactive art. Catalog essay for The Giver of Names exhibit at McDonald-Stewart Art Center

12. Mateas M, Stern A (2003) Facade: an experiment in building a fully-realized interactive drama. In: Game developer's conference: game design track, San Jose
13. McCorduck P (1991) Aaron's code: meta-art, artificial intelligence, and the work of Harold Cohen. W.H. Freeman and Company, New York

14. Lioret A (2005) Being paintings in proceedings of the ACM SIGGRAPH 05 electronic art and animation catalog. ACM, Los Angeles

15. Huang J (2005) Interactive wallpaper in proceedings of the ACM SIGGRAPH 05 electronic art and animation catalog. ACM, Los Angeles

16. Utterback C (2004) Untitled 5. SIGGRAPH 2005 Art show, Los Angeles

17. Viegas FB et al (2004) Artifacts of the presence era: using information visualization to create an evocative Souvenir. In: Proceedings of IEEE information visualization, Austin, pp 105111

18. Redstrom J, Skog T, Hallanas L (2000) Informative art: using amplified artworks as information displays. In: Proceedings of DARE. ACM, New York

19. Ishii H, Wisenski C, Brave S, Dahley A, Gorbet M, Ullmer B, Yarin P (1998) AmbientROOM: integrating ambient media with architectural space, $\mathrm{CHI} 98$ conference summary on human factors in computing systems, April 18-23, Los Angeles, pp 173174

20. Kidd C et al (1999) The aware home: a living laboratory for ubiquitous computing research. In: Proceedings of the second international workshop on cooperative buildings-CoBuild'99

21. Fitzpatrick G, Tolone WJ, Kaplan SM (1995) Work, locales and distributed social worlds. In: Proceedings of the fourth European conference on computer-supported cooperative work, pp $1-16$

22. Sonka M, Hlavec V, Boyle R (1999) Image processing analysis, and machine vision. PWS Publishing, Boston

23. Glasser B, Strauss A (1967) The discovery of grounded theory. Aldine, Chicago

24. Victor Shklovsky (1965) Art as technique. In: Lemon LT, Reis MJ (eds) Russian formalist criticism: four essays. University of Nebraska Press, Lincoln, pp 3-24

25. Bell G, Blythe M, Sengers P (2005) Making by making strange: defamiliarization and the design of domestic technologies. ACM Trans Comput Hum Interact 12(2):149-173

26. Krause J (2002) Color index. In: Warmke C (ed) Complete index. F\&W Publications, Cincinnati

27. Newell A (1993) Reflections on the knowledge level. Artif Intell 59(1-2):31-38 